Pacific Journal of Mathematics

ON ARITHMETIC PROPERTIES OF THE TAYLOR SERIES OF 


\title{
ON ARITHMETIC PROPERTIES OF THE TAYLOR SERIES OF RATIONAL FUNCTIONS, II
}

\author{
David G. CANTOR
}

\begin{abstract}
Suppose $a_{n}, b_{n}$, and $c_{n}=a_{n} b_{n}$ are sequences of algebraic integers and that all $b_{n}$ are nonzero. It is easy to verify that if both $a(z)=\sum_{n=0}^{\infty} a_{n} z^{n}$ and $b(z)=\sum_{n=0}^{\infty} b_{n} z^{n}$ are rational functions, then so is $c(z)=\sum_{n=0}^{\infty} c_{n} z^{n}$. We are interested in studying the conjecture that if $b(z)$ and $c(z)$ are rational functions, then so is $a(z)$. We shall prove this in the case that $b(z)$ has no more than three distinct singularities.
\end{abstract}

Let $k$ be an algebraic number field; denote by $M_{k}$ the set of valuations of $k$, normalized so as to satisfy the Artin product-formula. We assume, whenever convenient, that each valuation in $M_{k}$ has been extended in some fashion to $\Omega$, the algebraic closure of $k$. Let $S$ be a finite subset of $M_{k}$ containing all Archimedean valuations. We say that $\alpha \in k$ is an $S$-integer if $|\alpha|_{v} \leqq 1$ for all $v \in M_{k}-S$ and that $\alpha$ is an $S$-unit if $\alpha$ and $1 / \alpha$ are both $S$-integers. Let $a_{n}$ be a sequence of $S$ integers of $k$. Suppose there exist rational functions $b(z)=\sum_{n=0}^{\infty} b_{n} z^{n}$ and $c(z)=\sum_{n=0}^{\infty} c_{n} z^{n}$ whose coefficients lie in an extension field $K$ (possibly transcendental) of $k$; suppose that none of the $b_{n}$ are 0 and that $a_{n}=c_{n} / b_{n}$ for $n \geqq 0$. In [1], I showed that if $b(z)$ has only one singularity (possibly a pole of high multiplicity) then $a(z)=\sum_{n=0}^{\infty} a_{n} z^{n}$ is a rational function. In [6] G. Pathiaux extended this result by showing that, under the additional assumption that $K$ is algebraic, if $b(z)$ has at most two distinct singularities, then $a(z)$ is rational.

Here we shall study various extensions of these results. In particular we shall show that if $b(z)$ has at most three distinct singularities, then $a(z)$ is rational.

We note that since $b(z)$ and $c(z)$ are rational functions, we may write $b_{n}$ and $c_{n}$ as exponential polynomials:

$$
\begin{aligned}
& b_{n}=\sum_{i=1}^{r} \lambda_{i}(n) \theta_{i}^{n} \\
& c_{n}=\sum_{i=1}^{s} \mu_{i}(n) \varphi_{i}^{n}
\end{aligned}
$$

for all sufficiently large $n$. Here the $\lambda_{i}(n)$ and $\mu_{i}(n)$ are polynomials in $n$. By appropriately enlarging $K$, if necessary, we may assume that the $\theta_{i}$, the $\varphi_{i}$, and the coefficients of the polynomials $\lambda_{i}(n)$ and $\mu_{i}(n)$ all lie in $K$. By omitting a finite number of terms from each of the sequences $a_{n}, b_{n}, c_{n}$ we may assume that (1) and (2) hold for all $n \geqq 0$. The purpose of the first lemma is to show that we may 
assume that $K$ is algebraic over $h$.

Lemma 1. Suppose $a_{n}, b_{n}, c_{n}$ are sequences as above. There exist sequences $\bar{b}_{n}, \bar{c}_{n}$ lying in a finite algebraic extension of $k$ with $\bar{b}(z)=$ $\sum_{n=0}^{\infty} \bar{b}_{n} z^{n}$ and $\bar{c}(z)=\sum_{n=0}^{\infty} c_{n} z^{n}$ rational functions such that $a_{n} \bar{b}_{n}=\bar{c}_{n}$ for all integral $n \geqq 0$ and such that only finitely many $\bar{b}_{n}$ are 0 .

Proof. As above we may write $b_{n}=\sum_{i=1}^{r} \lambda_{i}(n) \theta_{i}^{n}$ and $c_{n}=$ $\sum_{i=1}^{s} \mu_{i}(n) \varphi_{i}^{n}$.

If all the coefficients of the $\lambda_{i}$ and the $\mu_{i}$, and the $\theta_{i}$ and $\varphi_{i}$ are in $k$, then the Lemma is true with the $\bar{b}_{n}=b_{n}$ and $\bar{c}_{n}=c_{n}$. We henceforth assume this not the case. Let $R$ be the ring generated by adjoining the $\theta_{i}$, the $\varphi_{i}$, the ratios $\theta_{i} / \theta_{j}$, and the coefficients of the $\lambda_{i}$ and the $\mu_{i}$ to $k$. By the assumption above the transcendence degree $t$ of $R / k$ is $\geqq 1$. We are going to construct a homomorphism $\tau$ of $R$ into a finite algebraic extension of $k$ such that $\tau$, when restricted to $k$, will be the identity. If $\tau \alpha$ is abbreviated $\bar{\alpha}$ then $\bar{b}_{n}=\sum_{i=1}^{r} \bar{\lambda}_{i}(n) \bar{\theta}_{i}^{n}$ and thus $\sum \bar{b}_{n} z^{n}$ is rational; similarly $\sum \bar{c}_{n} z^{n}$ is rational and since $a_{n} b_{n}=c_{n}$ clearly $a_{n} \bar{b}_{n}=\bar{c}_{n}$. The remainder of this proof is devoted to constructing such a homomorphism $\tau$ for which only finitely many $\bar{b}_{n}$ are zero. By the Noether normalization lemma [3], there exists a transcendence basis $x=\left(x_{1}, x_{2}, \cdots, x_{t}\right)$ for $R / k$ such that each element of $R$ is integral over $k[x]$. Since $R / k[x]$ is algebraic and finitely generated, its degree $d$ is finite. Each element $\alpha$ in $R$ satisfies a polynomial equation $f(\alpha)=0$, where

$$
f(Y)=\sum_{i=0}^{\infty} p_{i}(x) Y^{e-i}
$$

is a polynomial with coefficients $p_{i}(x)$ in $k[x]$, of degree $e \leqq d$, and monic $\left(p_{0}(x) \equiv 1\right)$. Any homomorphism $\tau$ of $k[x]$ into $k$, which is the identity on $k$, has the form $p(x) \rightarrow p(u)$ where $u=\left(u_{1}, u_{2}, \cdots, u_{t}\right)$ is a $t$-tuple of elements of $k$ and $p(x)$ is in $k[x]$. Such a homomorphism $\tau$ can be extended to a homomorphism of $R$ into $\Omega$, the algebraic closure of $k$ [3]. The image $\bar{\alpha}$ of $\alpha$ will satisfy the monic polynomial $\sum_{i=0}^{e} p_{i}(u) Y^{e-i}$ and hence have degree $\leqq e$ over $k$. Since $e \leqq d$, every element in $\tau R$ will have degree $\leqq d$ over $k$ and hence $\tau R$ will be contained in a finite algebraic extension of $k$. Moreover if $p_{e}(u) \neq 0$, then $\bar{\alpha} \neq 0$. Denote by $\Phi_{k}(h)$ the degree of the field generated by the primitive $h^{\text {th }}$ roots of unity over $k$. It is easy to verify that $\Phi_{k}(h) \geqq \Phi_{Q}(h) /[k: Q]$ where $Q$ is the field of rational numbers and $\Phi_{Q}(h)$ is, of course, Euler's phifunction. Since $\Phi_{Q}(h) \rightarrow \infty$ as $h \rightarrow \infty$, so does $\Phi_{k}(h)$. Let $h$ be the largest integer for which $\Phi_{k}(h) \leqq d$. Let $m$ be the least common multiple of all of the orders of all of the roots of unity which can be written in the form $\theta_{i} / \theta_{j}$. We can write 


$$
b_{m n+s}=\sum_{i=1}^{q} \eta_{i s}(n) \sigma_{i}^{n}
$$

where the $\sigma_{i}$ are the distinct $m^{\text {th }}$ powers of the $\theta_{i}$, and the $\eta_{i s}(n)$ are polynomials, not all 0 (for each value of $s$ ). Let $\alpha$ be the product of all the nonzero coefficients of the $\eta_{i s}(n)$ and the elements $\left(\sigma_{i} / \sigma_{j}\right)^{h !}-1$ for $i \neq j$ (the latter quantities are not 0 since the ratios $\sigma_{i} / \sigma_{j}$ cannot be roots of unity). Now let $u=\left(u_{1}, u_{2}, \cdots, u_{t}\right)$ be elements of $h$ for which $p_{e}(u) \neq 0$. Then under the homomorphism $\tau$, defined above, $\bar{\alpha}=\tau \alpha$ will be nonzero, and $\bar{\eta}_{i s}(n)$ (the polynomial obtained by applying $\tau$ to each coefficient of the polynomial $\left.\eta_{i s}(n)\right)$ will be the zero-polynomial if and only if $\eta_{i s}(n)$ is the zero-polynomial. None of the ratios $\bar{\sigma}_{i} / \bar{\sigma}_{j}$, with $i \neq j$, are roots of unity, for since $\left(\bar{\sigma}_{i} / \bar{\sigma}_{j}\right)^{h !} \neq 1$, if $\bar{\sigma}_{i} / \bar{\sigma}_{j}$ were a root of unity, it would have to have order $>h$ and hence degree $>d$ over $k$; but the latter is not the case. If any of the $m$ sequences $\bar{b}_{m n+s}$ had infinitely many zeros then either all of the polynomials $\bar{\eta}_{i s}(n)$ would be zero or by a theorem of Mähler [4] and Lech [5] the zeros would be periodic, and two of the $\bar{\sigma}_{i}$ would have ratio a root of unity. Thus the sequence $b_{n}$ has only finitely many zeros.

Lemma 2. Suppose $a_{n}$ is a sequence of S-integers of $k$, that $a_{n}=$ $c_{n} / b_{n}$ where $b_{n}=\sum \lambda_{i}(n) \theta_{i}^{n}$ is never 0 and $c_{n}=\sum \mu_{i}(n) \varphi_{i}^{n}$; suppose the $\theta_{i}, \varphi_{i}$ and the coefficients of the $\lambda_{i}(n)$ and the $\mu_{i}(n)$ are integers of $k$. Suppose there exists a valuation $v_{0} \in S$ such that $\left|\theta_{1}\right|_{v_{0}}>\left|\theta_{i}\right|_{v_{0}}$ for $i \geqq 2$. Then $\sum_{n=0}^{\infty} a_{n} z^{n}$ is rational.

Proof. Elementary estimates show there exist $M>0$ and $R>0$ such that $\left|b_{n}\right|_{v}$ and $\left|c_{n}\right|_{v}$ are $\leqq M R^{n}$ for all $v \in S$ and $n \geqq 0$, and that $\left|b_{n}\right|_{v} \leqq 1$ for all $v \notin S$ and $n \geqq 0$. Since $\Pi_{v \in S}\left|b_{n}\right|_{v} \geqq 1$, if $w \in S$, then

$$
\left|\frac{1}{b_{n}}\right|_{w} \leqq \prod_{\substack{v \in S \\ v \neq w}}\left|b_{n}\right|_{v} \leqq M^{s-1} R^{(s-1) n}
$$

where $s$ is the cardinality of $S$. Then $\left|a_{n}\right|_{w}=\left|c_{n} / b_{n}\right|_{w} \leqq M^{s} R^{s n}$. It follows that $\sum_{n=0}^{\infty} a_{n} z^{n}$ has positive radius of convergence in $k_{w}$, the completion of $k$ under the valuation $w$. Let $\widetilde{k}_{w}$ be the algebraic closure of $k_{w}$ and assume that $w$ has been extended to $\widetilde{k}_{w}$. Let $R_{w}$ be the radius convergence of $a(z)=\sum_{n=0}^{\infty} a_{n} z^{n}$ in $k_{w}$. Then $a(z)$ is analytic in $\widetilde{k}_{w}$ for $|z|_{w}<R_{w}$. Now

or

$$
\lambda_{1}(n) \theta_{1}^{n} a_{n}=c_{n}-\sum_{i=2}^{r} \lambda_{i}(n) \theta_{i}^{n} a_{n}
$$

$$
\sum_{n=0}^{\infty} \lambda_{1}(n) a_{n} z^{n}=c\left(\frac{z}{\theta}\right)-\sum_{i=2}^{r} \lambda_{i}(n)\left(\frac{\theta_{i}}{\theta_{1}}\right)^{n} a_{n} z^{n} .
$$

In the field $\widetilde{k}_{v_{0}}$, the algebraic closure of $k_{v_{0}}$, the last equation expresses 
$\sum_{n=0}^{\infty} \lambda_{1}(n) a_{n} z^{n}$ as a rational function plus a sum of functions each meromorphic for $|z|_{v_{0}} \leqq \delta R_{v_{0}}$ where $\delta=\min _{i \geqq 2}\left|\theta_{1} / \theta_{i}\right|_{v_{0}}$ is $>1$. Thus by analytic extension $\sum_{n=0}^{\infty} \lambda_{1}(n) a_{n} z^{n}$ is meromorphic for $|z|_{v_{0}}<\delta R_{v_{0}}$. Repeated applications of the above transformation show that $\sum_{n=0}^{\infty} \lambda_{1}(n)^{j} a_{n} z_{w_{n}}$ is meromorphic for $|\boldsymbol{z}|_{v_{0}}<\delta^{j} R_{v_{0}}$. Elementary estimates show that $\sum_{n=0}^{\infty} \lambda_{1}(n)^{j} a_{n} z^{n}$ has radius of convergence $R_{v}$ for all $v \in S$. Choosing $j$ so large that $\delta^{j} \prod_{v \in S} R_{v}$ is $>1$, we find, by a theorem of Dwork [2], that $\sum_{n=0}^{\infty} \lambda_{1}(n)^{j} a_{n} z^{n}$ is a rational function. By [1] so is $\sum_{n=0}^{\infty} a_{n} z^{n}$.

Lemma 3. Suppose $a_{n}$ is a sequence of S-integers of $k$, that $a_{n}=$ $c_{n} / b_{n}$ where $b_{n}=\sum \lambda_{i}(n) \theta_{i}^{n}$ is never zero and $c_{n}=\sum \mu_{i}(n) \varphi_{i}^{n}$, suppose the $\theta_{i}, \varphi_{i}$ and the nonzero coefficients of the $\lambda_{i}(n)$ and the $\mu_{i}(n)$ are $S$-units of $h$. Suppose there exists a valuation $v_{0}$ of $h$ such that $\left|\theta_{1}\right|_{v_{0}}<$ $\left|\theta_{i}\right|_{v_{0}}$ for $i \geqq 2$. Then $\sum_{n=0}^{\infty} a_{n} z^{n}$ is rational.

Poof. Extend the definition of $c_{n}$ and $b_{n}$ to negative $n$ by their formulas. If infinitely many such $b_{n}$ were zero, then by a theorem of Lech [4] and Mähler [5], $b_{n}$ would be zero for all $n$ in a doubly infinite arithmetic progression, contradicting the hypotheses. Extend the definition of $a_{n}$ to negative $n$ by putting $a_{n}=c_{n} / b_{n}$ if $b_{n} \neq 0$ and otherwise put $a_{n}=0$. Now let $v$ be any valuation of $M_{k}$ not in $S$. Then $v$ is an extension of a $p$-adic valuation $\mid l_{p}$ of $Q$. There exists an integer $f$ such that if $\alpha \in k$ and $|\alpha|_{v}=1$ then $\left|\alpha^{p^{f}}-1\right|_{v}<1$. Letting $m$ be an integer of the form $p^{h}\left(p^{f}-1\right)$, where $h$ is large, we find that $\left|\alpha^{m}-1\right|_{v}$ can be made very small. In particular we can choose $m$ so large that if $b_{n} \neq 0$ then $\left|b_{n+m}\right|_{v}=\left|b_{n}\right|_{v}$ and that $\left|c_{n+m}-c_{n}\right|_{v}<$ $\left|b_{n}\right|_{v}$. We can choose $m$ so large that $m+n \geqq 0$ and then $\left|c_{m+n} / b_{m+n}\right|_{v} \leqq 1$. Thus $\left|a_{n}\right|_{v} \leqq 1$. Restating all this, we have shown that there exists $n_{0}$ such that if $n \leqq n_{0}$ then $b_{n} \neq 0$ and if $v \in S$ then $\left|a_{n}\right|_{v} \leqq 1$. We apply Lemma 2 to the sequences $a_{n}^{\prime}=a_{n_{0}-n}, b_{n}^{\prime}=b_{n_{0}-n}$ and $c_{n}^{\prime}=c_{n_{0}-n}$, to conclude that $\sum_{n=0}^{\infty} a_{n}^{\prime} z^{n}$ is rational. It follows that $a_{n}$ can be written in the form

$$
a_{n}=\sum_{i=1}^{t} \eta_{i}(n) \sigma_{i}^{n}
$$

for $n \leqq n_{0}$. Then the exponential polynomial

$$
\sum_{i=1}^{s} \mu_{i}(n) \varphi_{i}^{n}-\sum_{i=1}^{n} \lambda_{i} \theta_{i}^{n} \sum_{i=1}^{t} \eta_{i}(n) \sigma_{i}^{n}
$$

is 0 for $n \leqq n_{0}$. By the theorem of Mähler [5] and Lech [4], it is identically 0 . Thus $\alpha_{n}=\sum_{i=1}^{t} \eta_{i}(n) \sigma_{i}^{n}$ for $n \geqq 0$ and $a(z)=\sum_{n=0}^{\infty} a_{n} z^{n}$ is a rational function.

We now come to the result mentioned at the beginning of this 
paper.

THEOREM 4. Suppose $a_{n}$ is a sequence of S-integers of $k$ and that $b_{n}$ and $c_{n}$ are sequences of elements of an extension field $K$ of $k$ such that $\sum_{n=0}^{\infty} b_{n} z^{n}$ and $\sum_{n=0}^{\infty} c_{n} z^{n}$ are rational functions and $b_{n}$ is never zero. If $a_{n}=c_{n} / b_{n}$ and the rational function $\sum_{n=0}^{\infty} b_{n} z^{n}$ has at most 3 distinct singularities then $\sum_{n=0}^{\infty} a_{n} z^{n}$ is rational.

Proof. By Lemma 1, we may assume $K$ is algebraic over $k$ and that $b_{n}=\sum_{i=1}^{r} \lambda_{i}(n) \theta_{i}^{n}$ and that $c_{n}=\sum_{i=1}^{s} \mu_{i}(n) \varphi_{i}^{n}$ where the $\theta_{i}, \varphi_{i}$ and all coefficients of the $\lambda_{i}$ and $\mu_{i}$ are algebraic over $k_{\text {. }}$ By replacing $k$ by a larger field and $S$ by the set of extensions of the valuations in $S$ to this new field, we may assume that the above quantities are, in fact, in $k$. By increasing $S$ appropriately, we may assume that those of the above quantities which are not zero are $S$-units. Now if $r=1$, the theorem follows immediately from [1]. If $r=2$ then either $\theta_{1} / \theta_{2}$ is a root of unity, in which case the theorem follows from the case $r=1$ or there is a valuation $v$ such that $\left|\theta_{1}\right|_{v}>\left|\theta_{2}\right|_{v}$, and the theorem follows from Lemma 2. If $r=3$ then either $\left|\theta_{1}\right|_{v}=\left|\theta_{2}\right|_{v}=\left|\theta_{3}\right|_{v}$, for all $v \in S$ and $\theta_{1} / \theta_{2}$ and $\theta_{1} / \theta_{3}$ are roots of unity, so the theorem follows from the case $r=1$, or there is a valuation $v_{0} \in S$ for which not all of the three values are equal. In the latter case we may assume that $\left|\theta_{1}\right|_{v_{0}} \leqq\left|\theta_{2}\right|_{v_{0}} \leqq\left|\theta_{3}\right|_{v_{0}}$ and $\left|\theta_{1}\right|_{v_{0}}<\left|\theta_{3}\right|_{v_{0}}$. If $\left|\theta_{2}\right|_{v_{0}}=\left|\theta_{3}\right|_{v_{0}}$ then the theorem follows from Lemma 3 , and otherwise from Lemma 2.

It is worth noting that the method of the theorem cannot be extended to the case where $b(z)$ has 4 singularities. In fact, consider the case where $k$ is the field $Q(i)$ where $i=\sqrt{-1}$ and $\theta_{1}=(1+2 i) \times$ $(1+4 i), \theta_{2}=(1+2 i)(1-4 i), \theta_{3}=(1-2 i)(1+4 i), \theta_{4}=(1-2 i)(1-4 i)$. The ideals generated by $(1+2 i),(1-2 i),(1+4 i),(1-4 i)$ are prime and give rise to 4 valuations of $Q(i)$. At each of these valuations, two of the $\theta_{j}$ take one value and two another. For example at the valuation corresponding to the prime ideal generated by $1-2 i, \theta_{1}$ and $\theta_{2}$ both have value 1 , while $\theta_{3}$ and $\theta_{4}$ both have the same value which is less than 1. All $4 \theta_{j}$ take the same value at all other valuations. Thus the hypotheses of Lemma 2 or Lemma 3 cannot be met.

\section{REFERENCES}

1. D. G. Cantor, On arithmetic properties of the Taylor series of rational functions, Canad. J. Math., 21 (1969), 378-382.

2. B. Dwork, On the rationality of the zeta function of an algebraic variety, Amer.

J. Math., 82 (1960), 631-648.

3. S. Lang, Introduction to Algebraic Geometry, Interscience, New York, 1958.

4. C. Lech, $A$ note on recurring series, Arch. Mat., 2 (1953), 417-421.

5. K. Mähler, On the Taylor coefficients of rational functions, Proc. Cambridge Philos. 
Soc., 52 (1956), 39-48.

6. G. Pathiaux, Algébre de Hadamard de fractions rationnelles, C. R. Acad. Sci., Paris Ser. A-B, 267 (1968), A977-A979.

Received February 12, 1971. This research was supported in part by the Sloan Foundation and National Science Foundation Grant \#GP-23113.

University of California, Los ANgeles 


\section{PACIFIC JOURNAL OF MATHEMATICS}

\section{EDITORS}

\author{
H. SAMELSON \\ Stanford University \\ Stanford, California 94305

\section{R. HoвBY} \\ University of Washington \\ Seattle, Washington 98105
}

\section{J. DugundJI}

Department of Mathematics University of Southern California Los Angeles, California 90007

\author{
RichaRd ARENS \\ University of California \\ Los Angeles, California 90024
}

\section{ASSOCIATE EDITORS}
E. F. BECKENBACH
B. H. NEUMANN
F. WOLF
K. YoshidA

\section{SUPPORTING INSTITUTIONS}

\author{
UNIVERSITY OF BRITISH COLUMBIA \\ CALIFORNIA INSTITUTE OF TECHNOLOGY \\ UNIVERSITY OF CALIFORNIA \\ MONTANA STATE UNIVERSITY \\ UNIVERSITY OF NEVADA \\ NEW MEXICO STATE UNIVERSITY \\ OREGON STATE UNIVERSITY \\ UNIVERSITY OF OREGON \\ OSAKA UNIVERSITY
}

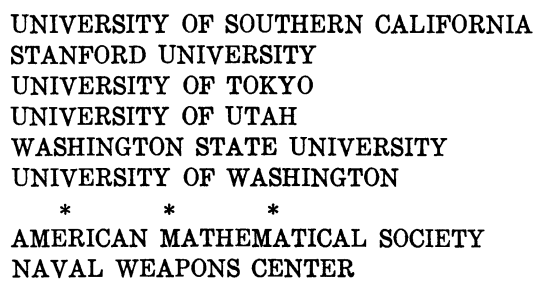

The Supporting Institutions listed above contribute to the cost of publication of this Journal, but they are not owners or publishers and have no responsibility for its content or policies.

Mathematical papers intended for publication in the Pacific Journal of Mathematics should be in typed form or offset-reproduced, (not dittoed), double spaced with large margins. Underline Greek letters in red, German in green, and script in blue. The first paragraph or two must be capable of being used separately as a synopsis of the entire paper. The editorial "we" must not be used in the synopsis, and items of the bibliography should not be cited there unless absolutely necessary, in which case they must be identified by author and Journal, rather than by item number. Manuscripts, in dup icate if possible, may be sent to any one of the four editors. Please classify according to the scheme of Math. Rev. Index to Vol. 39. All other communications to the editors should be addressed to the managing editor, Richard Arens, University of California, Los Angeles, California, 90024.

50 reprints are provided free for each article; additional copies may be obtained at cost in multiples of 50 .

The Pacific Journal of Mathematics is published monthly. Effective with Volume 16 the price per volume (3 numbers) is $\$ 8.00$; single issues, $\$ 3.00$. Special price for current issues to individual faculty members of supporting institutions and to individual members of the American Mathematical Society: $\$ 4.00$ per volume; single issues $\$ 1.50$. Back numbers are available.

Subscriptions, orders for back numbers, and changes of address should be sent to Pacific Journal of Mathematics, 103 Highland Boulevard, Berkeley, California, 94708.

PUBLISHED BY PACIFIC JOURNAL OF MATHEMATICS, A NON-PROFIT CORPORATION

Printed at Kokusai Bunken Insatsusha (International Academic Printing Co., Ltd.), 270, 3-chome Totsuka-cho, Shinjuku-ku, Tokyo 160, Japan. 


\section{Pacific Journal of Mathematics}

\section{Vol. 41, No. 2 December, 1972}

Tom M. (Mike) Apostol, Arithmetical properties of generalized Ramanujan sums .......................................... 281

David Lee Armacost and William Louis Armacost, On p-thetic groups ........ 295

Janet E. Mills, Regular semigroups which are extensions of groups .......... 303

Gregory Frank Bachelis, Homomorphisms of Banach algebras with minimal ideals ................................................ 307

John Allen Beachy, A generalization of injectivity .................. 313

David Geoffrey Cantor, On arithmetic properties of the Taylor series of rational functions. II.........................................

Václáv Chvátal and Frank Harary, Generalized Ramsey theory for graphs. III.

Small off-diagonal numbers .................................. 335

Frank Rimi DeMeyer, Irreducible characters and solvability of finite groups . . . . 347

Robert P. Dickinson, On right zero unions of commutative semigroups........ 355

John Dustin Donald, Non-openness and non-equidimensionality in algebraic

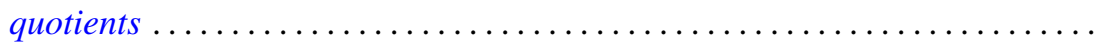

John D. Donaldson and Qazi Ibadur Rahman, Inequalities for polynomials with a prescribed zero ........................................ 375

Robert E. Hall, The translational hull of an $N$-semigroup ................ 379

John P. Holmes, Differentiable power-associative groupoids.............. 391

Steven Kenyon Ingram, Continuous dependence on parameters and boundary data for nonlinear two-point boundary value problems .

Robert Clarke James, Super-reflexive spaces with bases ..........

Gary Douglas Jones, The embedding of homeomorphisms of the plane in

continuous flows...............................

Mary Joel Jordan, Period $H$-semigroups and $t$-semisimple periodic

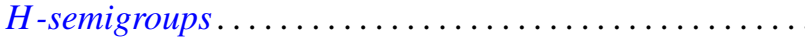

Ronald Allen Knight, Dynamical systems of characteristic 0

Kwangil Koh, On a representation of a strongly harmonic ring by sheaves...

Hui-Hsiung Kuo, Stochastic integrals in abstract Wiener space. ..

Thomas Graham McLaughlin, Supersimple sets and the problem of extending a

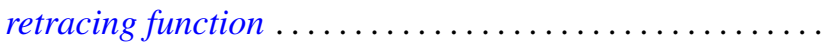

William Nathan, Open mappings on 2-manifolds .

M. J. O'Malley, Isomorphic power series rings

Sean B. O'Reilly, Completely adequate neighborhood systems and metrization

Qazi Ibadur Rahman, On the zeros of a polynomial and its derivative...

Russell Daniel Rupp, Jr., The Weierstrass excess function ..

Hugo Teufel, A note on second order differential inequalities and functional

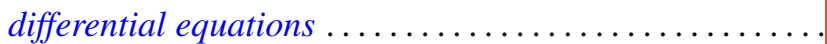

M. J. Wicks, A general solution of binary homogeneous equations over free 